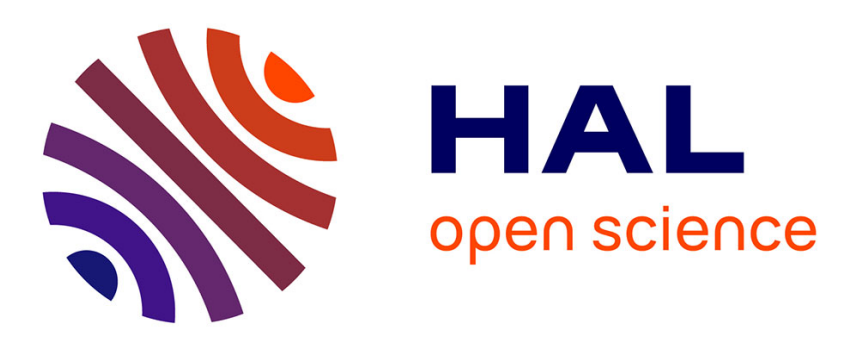

\title{
Drastic changes in the electronic and magnetic structures of hydrogenated U2Ti intermetallic from first principles
}

Samir F. Matar

\section{- To cite this version:}

Samir F. Matar. Drastic changes in the electronic and magnetic structures of hydrogenated U2Ti intermetallic from first principles. Journal of Magnetism and Magnetic Materials, 2014, 358-359, pp.70-75. 10.1016/j.jmmm.2014.01.003 . hal-00956569

\section{HAL Id: hal-00956569 https://hal.science/hal-00956569}

Submitted on 21 Mar 2014

HAL is a multi-disciplinary open access archive for the deposit and dissemination of scientific research documents, whether they are published or not. The documents may come from teaching and research institutions in France or abroad, or from public or private research centers.
L'archive ouverte pluridisciplinaire HAL, est destinée au dépôt et à la diffusion de documents scientifiques de niveau recherche, publiés ou non, émanant des établissements d'enseignement et de recherche français ou étrangers, des laboratoires publics ou privés. 
Drastic changes in the electronic and magnetic structures of hydrogenated $\mathrm{U}_{2} \mathrm{Ti}$ intermetallic from first principles.

Samir F. Matar ${ }^{\mathrm{a}, \mathrm{b}, *}$

${ }^{\text {a }}$ CNRS, ICMCB, UPR 9048, F-33600 Pessac, France

${ }^{\mathrm{b}}$ Univ. Bordeaux, ICMCB, UPR 9048, F-33600 Pessac, France

*Email: matar@icmcb-bordeaux.cnrs.fr

Keywords: Intermetallic. Uranium. Magnetism. Bonding. Interstitial content. DFT. Electronic structure calculation

Abstract

Based on DFT calculations, hydrogen insertion in $U_{2} T$ i intermetallic with $A_{1} B_{2}$-type structure leading to $\mathrm{U}_{2} \mathrm{TiH}_{3}$ with sites identified from energy discrimination between different hypotheses is found to decrease the cohesive energy through weakening of the Ti-U bonds, to expand the lattice along $c$ hexagonal axis due to strong $U-U$ in-plane bonding and to induce iono-covalent character with $H^{-0.65}$. The anisotropic volume expansion involves a strong localization of out-of-plane uranium $f_{z-3 z r}^{3}$ orbitals leading to developing a magnetic moment on uranium in a predicted ferromagnetic ground state, oppositely to non magnetic intermetallic.

Keywords. Uranium intermetallics. Hydrides. Magnetism. DFT. AIM. ASW. PAW-GGA 


\section{Introduction}

Uranium and its alloys are known to uptake considerable amounts of hydrogen. Uranium trihydride $\mathrm{UH}_{3}$ is a good candidate for hydrogen absorption and release and could be envisaged for the use of depleted uranium produced from nuclear waste were it not for its high pyrophoricity caused by the hydrogen induced disintegration into fine powders through the 'decrepitating process' [1]. However when alloyed with other elements as $T=\mathrm{Ti}$ or $\mathrm{Zr}$ the binary alloys such as $U T, U T_{2}$ and $U_{2} T$ have been found to present better resistance to decrepitating while absorbing large amounts of hydrogen up to $7 \mathrm{H}$ per formula unit (FU) [2].

Introducing hydrogen in the intermetallic structure has been shown to induce physical and chemical effects pertaining to the expanded lattice and to the interaction of hydrogen with the atomic constituents [3]. Whereas metallic uranium is not magnetically ordered, the absorption of hydrogen leads to significant changes of the magnetic properties with the onset of ferromagnetic ordering at $T_{C}=10 \mathrm{~K}$ and a magnetization of $\mathrm{M}=1.45 \mu_{\mathrm{B}}$ in deuterated $\mathrm{UH}_{3}\left(\mathrm{UD}_{3}\right)$ [4]. In fact, except for uranium heavy Fermion and superconductor compounds, uranium $5 f$ states act in many itinerant intermetallic compounds like transition metal $d$ states [5].

The lattice expansion induced by inserting non metals like $\mathrm{H}$ involves two major effects: a 'negative pressure' leading to a larger separation between the atomic constituents with subsequent localization of the valence states on one hand and the chemical bonding between the metals and hydrogen valence states on the other hand [6]. In this context the binaries $U_{2} T$ ( $T$ $=\mathrm{Ti}, \mathrm{Zr}$ ) and their hydrogenated homologues present a particularly interesting case: The structure is based on the hexagonal $\mathrm{AlB}_{2}$-type (Fig. 1) and the intermetallic uptakes up to 4 $\mathrm{H} / \mathrm{FU}$ without reporting symmetry changes [2]. This is less than the cubic C15 Laves UT 2 compounds which can absorb up to $7 \mathrm{H} / \mathrm{FU}$. Whereas the $\mathrm{H}$ (deuterium) atomic sites within $\mathrm{UT}_{2} \mathrm{H}_{\mathrm{x}}$ Laves phases were determined experimentally [7], to the best of our knowledge no experimental hydrogen positions for $\mathrm{U}_{2} T \mathrm{H}_{\mathrm{x}}$ are available.

Recently the electronic structure and the elastic properties of $\mathrm{U}_{2} \mathrm{Ti}$ were investigated by Hasan et al. [8] within the well established density functional quantum theoretical DFT framework [9]. Focusing on this binary intermetallic we investigate within the same theoretical framework the electronic and magnetic structure of the hydrogenated homologue comparatively with the pristine intermetallic by proposing the most favorable hydrogen insertion sites discriminated from cohesive energies among different working hypotheses. The optimized ternary hydride is then examined for the change of cohesive and mechanical properties with respect to pristine 
intermetallic. Subsequent characterizations of the electronic and magnetic structures and chemical bonding properties with all electrons calculations are carried out.

\section{Crystal structure.}

The hexagonal structure of $\mathrm{U}_{2} \mathrm{Ti}$ is $\mathrm{AlB}_{2}$-type (space group $\mathrm{P} 6 / \mathrm{mmm}$ ), one FU per cell (Fig. 1 top) with Ti at $1 a(0,0,0)$ and pairs of $U$ at $2 d(1 / 3,2 / 3,1 / 2)$ with $d_{U-U} \sim 2.6 \AA$. In an extended $a, b$ plane, the U-U pairs arrange in hexagons with a two-dimensional network of honeycomb layers stacked perpendicularly to the hexagonal $c$ axis. (cf. Fig. 1 bottom).

Different hypotheses can be envisaged for hydrogen insertion. They belong to two major classes pertaining to placing $\mathrm{H}$ either at $z=0$ (Wyckoff positions $2 c, 3 f, 6 j, 6 /$ ) or $z=1 / 2$ (Wyckoff positions $2 d, 3 g, 6 k, 6 m)$. Intermediate $z$ have been also envisaged with $4 h$ positions $(1 / 3,2 / 3, z)$ and four inserted hydrogen atoms.

\section{Computational methodology}

Two computational methods within the DFT were used in a complementary manner. The Vienna ab initio simulation package (VASP) code $[10,11]$ allows geometry optimization and total energy calculations. For this we use the projector augmented wave (PAW) method $[11,12]$, built within the generalized gradient approximation (GGA) scheme following Perdew, Burke and Ernzerhof (PBE) [13]. Preliminary calculations with local density approximation (LDA) [14] led to largely underestimated volumes versus experiment. The conjugate-gradient algorithm [15] is used in this computational scheme to relax the atoms. The tetrahedron method with Blöchl corrections [12] as well as a Methfessel-Paxton [16] scheme were applied for both geometry relaxation and total energy calculations. Brillouin-zone (BZ) integrals were approximated using special $\boldsymbol{k}$-point sampling. The optimization of the structural parameters was performed until the forces on the atoms were less than $0.02 \mathrm{eV} / \AA ̊ \AA$ and all stress components less than $0.003 \mathrm{eV} / \AA^{3}$. The calculations are converged at an energy cut-off of $400 \mathrm{eV}$ for the plane-wave basis set with respect to the $\boldsymbol{k}$-point integration with a starting mesh of $4 \times 4 \times 4$ up to $8 \times 8 \times 8$ for best convergence and relaxation to zero strains. The calculations are scalar relativistic. From the results we derive the trends of charge transfers through the analysis of the charge density issued from the self consistent calculations using the AIM (atoms in molecules theory) approach [17].

Using the optimized geometry for the most stable hydrogenated $\mathrm{U}_{2} \mathrm{Ti}$ configuration identified from energy criteria, scalar relativistic all-electrons calculations within the GGA were carried out for a full 
description of the electronic and magnetic structure and the properties of chemical bonding, using the full potential scalar-relativistic augmented spherical wave (ASW) method [18, 19]. In the minimal ASW basis set, we chose the outermost shells to represent the valence states and the matrix elements were constructed using partial waves up to $I_{\max }+1=4$ for $\mathrm{U}, I_{\max }+1=3$ for Ti and $I_{\max }+1=$ 1 for $\mathrm{H}$. Self-consistency was achieved when charge transfers and energy changes between two successive cycles were below $10^{-8}$ and $10^{-6} \mathrm{eV}$, respectively. BZ integrations were performed using the linear tetrahedron method within the irreducible wedge. Besides the site projected density of states, we discuss qualitatively the pair interactions based on the overlap population $\mathrm{S}_{\mathrm{ij}}$ analysis with the crystal orbital overlap population (COOP) [20]. In the plots, positive, negative, and zero COOP indicate bonding, anti-bonding, and non-bonding interactions, respectively.

\section{Results of geometry optimizations, cohesive energies, and charge analysis.}

With the purpose of evaluating the hydrogen insertion effects on the electronic structure of $\mathrm{U}_{2} \mathrm{Ti}$, two hypotheses were firstly considered by placing three $\mathrm{H}$ atoms at $z=0$ (Hypo.1: $\mathrm{H}$ at $3 \mathrm{f}$ ) and at $z=1 / 2$ (Hypo.2: $\mathrm{H}$ at $3 g$ ). Table 1 shows the results of geometry relaxation and the crystal and energy parameters. The calculated lattice constants for the binary intermetallic are found close to the computed values [8] and slightly smaller than the experiment with $\Delta \mathrm{V}=-2 \AA^{3}$.

Cohesive energies and charge transfers.

The cohesive energy is obtained from the total energy subtracted from the contributions of the individual atomic constituents in their ground state structures: $E(T i$ hex. $)=-7.21 \mathrm{eV} / \mathrm{at}$.; $E(\mathrm{U} \mathrm{bcc})$ $=-11.00 \mathrm{eV} /$ at., and for dihydrogen, $\mathrm{H} 2$, in a large cubic box, $E(\mathrm{H} 2)=-6.935 \mathrm{eV}$.

Then $E_{\text {coh. }}\left(\mathrm{U}_{2} \mathrm{Ti}\right)=E_{\text {Tot. }}\left(\mathrm{U}_{2} \mathrm{Ti}\right)-\{2 E(\mathrm{U})+E(\mathrm{Ti})\}=-1.382 \mathrm{eV} / \mathrm{FU}$. Negative energy values are indicative of stabilized configuration and expectedly the $\mathrm{U}_{2}$ Ti binary is stable. In this context it is interesting to compare the cohesive energy of $\mathrm{U}_{2} \mathrm{Ti}$ with those of $\mathrm{UTi}_{2}$ and UTi calculated in their ground state structures, cubic Laves and rocksalt respectively: $E_{\text {coh. }}\left(\mathrm{UTi}_{2}\right)=-0.48 \mathrm{eV} / \mathrm{FU}$ and $E_{\text {coh. }}(\mathrm{UTi})=-$ $0.30 \mathrm{eV} / \mathrm{FU}$. Clearly $\mathrm{U}_{2} \mathrm{Ti}$ is the most cohesive among the three binary intermetallic compounds and this result should be assigned to the particular atomic arrangement of uranium in the structure: the $U-U$ pairs condense in the long range into $U 6$ hexagons as shown in Fig. 1 . Also it can be predicted that such U-U bonds will be difficult to break such as by hydrogen insertions. In fact the optimized geometries for Hypo.1 and Hypo.2 point to quite different results: In Hypo.1, the lattice constant decreases slightly with respect to $\mathrm{U}_{2} \mathrm{Ti}$ whereas the $c / a$ ratio increases considerably leading to strong increase of $\mathrm{Ti}-\mathrm{U}$ interatomic distances ( $\mathrm{Ti}$ and $\mathrm{U}$ being in two different planes at $z=0$ and $z=1 / 2$ resp.). Oppositely a slight decrease of the $U$ - $U$ distance is 
observed due to the contraction of $a$. This leads to assume that the hexagonal U6 -like plane tolerates little modification so that the volume expansion operates in an anisotropic manner, i.e. along $c$.

The cohesive energy of the hydrogenated compound is obtained as follows:

$E_{\text {coh. }}\left(\mathrm{U}_{2} \mathrm{TiH}_{3}\right)=E_{\text {Tot. }}\left(\mathrm{U}_{2} \mathrm{TiH}_{3}\right)-\{2 E(\mathrm{U})+E(\mathrm{Ti})+3 / 2 E(\mathrm{H} 2)\}=-0.507 \mathrm{eV} / \mathrm{FU}$.

Hydrogen insertion leads to a large destabilization of the intermetallic by $\sim 3$. Nevertheless the cohesive energy remains negative and its magnitude suggests that hydrogen can be readily released from the ternary compound which exhibits an iono-covalent -like behavior versus fully covalent (metallic, delocalized electrons) $\mathrm{U}_{2} \mathrm{Ti}$.

Regarding Hypo.2 implying a breaking of the U-U bond ex. for $\mathrm{H}$ at $(1 / 21 / 21 / 2)$, the system is found completely destabilized with a reduced $c / a$ ratio and a calculated largely positive cohesive energy (Table 1 last column). The $\mathrm{U}-\mathrm{H}$ distance is short with almost half the value obtained in Hypo.1. The ternary in Hypo.2 configuration cannot be retained as a candidate.

For the sake of completeness other hypotheses were also considered. They equally led to positive cohesive energies:

$* 4 \mathrm{H}$ at $4 h$ positions $\pm(1 / 32 / 3 z): z_{\text {calc. }}=0.239, \mathrm{~d}_{\mathrm{U}-\mathrm{H}}=2.01 \AA$ and $E_{\mathrm{Tot}} .\left(\mathrm{U}_{2} \mathrm{TiH}_{4}\right)=-38.79 \mathrm{eV}$, leading to $E_{\text {coh. }}\left(\mathrm{U}_{2} \mathrm{TiH}_{4}\right)=+4.27 \mathrm{eV} / \mathrm{FU}$.

$* 2 \mathrm{H}$ at $2 c\left(\begin{array}{lll}1 / 3 & 2 / 3 & 0\end{array}\right)$ and $2 \mathrm{H}$ at $2 e\left(\begin{array}{ll}0 & \mathrm{z}\end{array}\right): z_{\text {calc. }}=0.378, \mathrm{~d}_{\mathrm{U}-\mathrm{H}}=2.31 \AA \mathrm{d}_{\mathrm{Ti}-\mathrm{H}}=1.75 \AA$; $E_{\mathrm{Tot}}\left(\mathrm{U}_{2} \mathrm{TiH}_{4}\right)=-$ $40.25 \mathrm{eV}$ leading to $E_{\text {coh. }}\left(\mathrm{U}_{2} \mathrm{TiH}_{4}\right)=+2.80 \mathrm{eV} / \mathrm{FU}$.

Then, considering the most stable configuration, with $\mathrm{H}$ at $(3 \mathrm{f})$ Wyckoff positions, the charge transfers obtained within AIM theory through an analysis of the charge density are such that they lead to neutrality:

$\mathrm{Q}(\mathrm{Ti})=+0.15 ; \mathrm{Q}(\mathrm{U})=+0.90$ and $\mathrm{Q}(\mathrm{H})=-0.65 ;$ and $\mathrm{Q}(\mathrm{Ti})+2 \mathrm{Q}(\mathrm{U})+3 \mathrm{Q}(\mathrm{H})=0$.

The charge transfer from the metallic elements leads to $\mathrm{H}^{-0.65}$ hydride anion. This is a charge indicating an "iono-covalent" character in so far that it is lower than in a largely ionic hydride as $\mathrm{MgH}_{2}$ where hydrogen carries a charge close to -0.83 (cf. [21] and therein refs.). Also a larger magnitude of charge transfer is observed from uranium to $\mathrm{H}$ versus Ti to $\mathrm{H}$. This is an indication of a significant bonding between the two atoms and it will be discussed further in the next section relating to the chemical bonding. 
Mechanical properties: Energy - Volume equations of state (EOS)

The geometry relaxation results can be further assessed from plotting the energy - volume $E_{i}$ $=f\left(V_{i}\right)$ curves for discrete sets of $V_{i}<V_{\text {opt. }}$ and $V_{i}>V_{\text {opt. }} ; V_{\text {opt. }}$ being the geometry optimized volume as given in Table 1. The $\mathrm{E}_{i}=f\left(\mathrm{~V}_{i}\right)$ curves are shown in Fig. 2 for the two phases. The energy and volume scales correspond to one formula unit (FU). The two curves exhibit quadratic shapes and they were fitted by a Birch-Murnaghan EOS [22] up to the $3^{\text {rd }}$ order expressed as follows:

$$
E(V)=E_{0}\left(V_{0}\right)+\frac{9}{8} V_{0} B_{0}\left[\left(V_{0} / V\right)^{2 / 3}-1\right]^{2}+\frac{9}{16} B_{0}\left(B^{\prime}-4\right) V_{0}\left[\left(V_{0} / V\right)^{2 / 3}-1\right]^{3}
$$

In this equation $E_{0}, V_{0}, B_{0}$ and $B^{\prime}$ are the equilibrium energy, the volume, the bulk modulus and its pressure derivative, respectively. The obtained values are displayed in the inserts of Fig. 2 . There is a better agreement with experiment for the volume magnitudes and the energies are close to those obtained from the geometry optimizations in Table 1. The pressure derivative of the bulk modulus $B^{\prime}$ is $\sim 4$, a value usually encountered. $\chi^{2}$ corresponds to the goodness of fit: the lower its value the better the fit; whence the reliable fit obtained with $\chi^{2} \sim 10^{-6}$. The bulk modulus $\mathrm{B}_{0}\left(\mathrm{U}_{2} \mathrm{Ti}\right)=134 \mathrm{GPa}$ is 9 GPa larger than the value obtained in a different manner by Hasan et al. from using the elastic constants [8]. While this difference can be due to the methods, i.e. EOS fit versus averaging over elastic constants, we underline the importance of comparing the $B_{\circ}$ magnitudes between the intermetallic and the hydrogenated compound. $\mathrm{B}_{0}$ of the latter is only slightly smaller by $2 \mathrm{GPa}$ whereas the volume has increased by $25 \%$ for $\mathrm{U}_{2} \mathrm{TiH}_{3}$. In as far as the bulk modulus reflects the resistance of the material to isotropic compression one would expect a largely compressible hydrogenated compound due to the significant volume increase. Then there should be another mechanism hindering the compression. In fact the negative pressure-like effect induced by hydrogen is partly compensated by the bonding between $\mathrm{H}$ and the metal substructures.

\section{All-electrons calculations}

Analyses of the density of states DOS and chemical bonding COOP.

Using the calculated structural data in Table 1, scalar relativistic calculations were carried out with the full potential ASW method for a detailed analysis of the atom resolved electronic density of states (PDOS) and the overlap population.

Starting from a spin degenerate non-magnetic configuration (NSP), Fig. 3 provides the site projected density of states (PDOS) in two panels corresponding to the intermetallic and to the hydrogenated compound. The energy along the $x$ axis is with respect to the Fermi level $\left(E_{\mathrm{F}}\right)$. The low fillings of Ti $(d)$ 
and $U(f)$ in the atomic states lead to the centering of $3 d$ and $5 f$ states above $E_{\mathrm{F}}$ within the conduction band $\mathrm{CB}$. In spite of this common feature observed for both compounds, large differences can be observed between them. This is particularly true in $\mathrm{U}_{2} \mathrm{Ti}$ for the broad shape of the DOS and the low magnitude density of states at $E_{\mathrm{F}},\left(n\left(E_{\mathrm{F}}\right)\right)$ especially for uranium. Oppositely $\mathrm{U}_{2} \mathrm{TiH}_{3}$ has a broader valence band (VB) by $\sim 2 \mathrm{eV}$ due to low energy lying H-PDOS thanks to their electronegative character and the PDOS are characterized by localized, sharp and more than 3 times larger $n\left(E_{\mathrm{F}}\right)$. An analysis of the $m_{l}$ components of the seven $f$ orbitals allows assigning the intense peak at $\mathrm{E}_{\mathrm{F}}$ to the out-of-plane $f_{z-3 z r}^{3}{ }^{2}$ with $I=3$, and $m_{l}=0$ depicted on the DOS panel. Then the expanded cell and the U-H interaction through the charge transfer leading to an iono-covalent compound are at the origin of the significant localization of some of the uranium $5 f$ states. In the framework of the Stoner theory of band ferromagnetism [23] this is indicative of a magnetic instability in such a spin degenerate configuration and a finite magnetic moment should develop mainly due to $f_{z-3 z r}^{3}$-like orbitals, while the other $f$ orbitals involved in the bonding within the $U 6$ ring will not polarize. In this context it is relevant to evoke the empirical Hill critical distance, $d_{U-u} \sim 3.5 \AA$ [24] below which there is, generally, no intra-band spin polarization because of an insufficient localization of the $U(5 f)$ band broadened due to the $f$ - $f$ direct overlap. This criterion is obeyed for the $f$ orbitals involved with the strong bonding within the $U 6$ hexagons (Fig. 1), and somehow not obeyed for the out-of-plane orbitals. The magnetic moment will be carried by these orbitals. Obviously this ternary compound is an illustration of structure $\Leftrightarrow$ property relationship.

Spin polarized (SP) calculations actually lead to a non magnetic configuration for $\mathrm{U}_{2} \mathrm{Ti}$ and to a total magnetization of $3.05 \mu_{\mathrm{B}} / \mathrm{FU}$ (spin-only) in a ferromagnetic configuration. The major contribution to total magnetization arises from uranium with $M(U)=1.59 \mu_{B}$ while $M(T i)=-0.02 \mu_{B}$; its negative small magnitude signals an induced character due to its bonding with uranium. The site and spin $(\uparrow, \downarrow)$ projected density of states shown in Fig. 4 mirror these results: In spin polarized calculations the magnetic moment arises from the shift between majority $\uparrow$ spin populations and minority $\downarrow$ spins ones through magnetic exchange. This is observed mainly for uranium $f$-PDOS around $\mathrm{E}_{\mathrm{F}}$ while $\mathrm{Ti}$ PDOS (as well as $\mathrm{H}$ ) exhibit small energy shift. The large $f$ PDOS peak is still observed at $\mathrm{E}_{\mathrm{F}}$ for $\uparrow$ spin but with more than half its magnitude in the NSP calculation (Fig. 3); also a minimum $\downarrow$ spins DOS magnitude is observed leading to the almost integer value of the total magnetization. With respect to the NSP configuration, the system stabilizes in the SP configuration by $-0.2 \mathrm{eV} / \mathrm{FU}$.

For the search of the actual magnetic ground state, an antiferromagnetic configuration was tested with a supercell created by doubling the $c$ parameter and dispatching the crystal into two magnetic subcells, one of them for $\Uparrow$ SPIN and the other for $\Downarrow$ SPIN. The result is zero magnetization as 
expected, and a raise of the energy by $+0.09 \mathrm{eV} / \mathrm{FU}$. Thus the magnetic ground state is predicted to be ferromagnetic. Lastly we note that the magnitude of magnetization only slightly changed with GGA+U calculations $(U=4 \mathrm{eV})$. This stresses furthermore the band magnetism behavior of $\mathrm{U}_{2} \mathrm{TiH}_{3}$, which was already visible from the large band widths seen in the DOS.

The similar PDOS shapes between the constituents in both panels signal the quantum mixing between the different valence basis states. In this context we analyze in the following paragraph the change of the inter-metal bonding from $\mathrm{U}_{2} \mathrm{Ti}$ to $\mathrm{U}_{2} \mathrm{TiH}_{3}$ on one and the magnitudes of the metal hydrogen bonding in the latter, on the other hand. The COOP criterion, based on the overlap matrix $\mathrm{S}_{\mathrm{ij}}$ is used. Fig. 5 shows in two panels the COOP for the metal-metal bonding. In $\mathrm{U}_{2} \mathrm{Ti}$ (upper panel) the major bonding magnitude is observed for $\mathrm{T}-\mathrm{U}$, followed by $\mathrm{U}-\mathrm{U}$ whereas the Ti-Ti bonding is weakest. These bonding magnitudes follow the course of the interatomic distances, $d_{U-T_{\mathrm{i}}}<d_{U-U}<d_{T i-T i}$. The positive COOP signaling bonding behavior is observed throughout the VB and extends above, within the $\mathrm{CB}$. Consequently the structure stability is ensured by $\mathrm{Ti}-\mathrm{U}$ bonding as well as by $\mathrm{U}-\mathrm{U}$ interactions to less extent. The bonding behavior observed in the lower panel is largely differentiated in shape, with more localized peaks especially in the neighborhood of the Fermi level, as well as with the lower magnitude $\mathrm{Ti}-\mathrm{U}$ bonding due to the larger separation between them. The $\mathrm{U}-\mathrm{U}$ bonding COOPs have a similar intensity as in the intermetallic albeit with different shapes. The overall metalmetal bonding intensity is larger in the intermetallic as with respect to the hydrogenated compound as we found upon integrating the curves. This reflects the cohesive energy differences between the two compounds with the largest stabilization of the binary intermetallic. At Fig. 4, the metalhydrogen COOPs are shown with an account of one metal atom and all three hydrogen atoms in order to enable for comparisons. The $\mathrm{U}-\mathrm{H}$ bonding is larger as the curve corresponding to Ti-H COOP is found underneath in its major part. This reflects the larger charge transfer from $\mathrm{U}$ to $\mathrm{H}$ versus Ti to H.

\section{Conclusions}

In this work ab initio results on the electronic structures of $\mathrm{U}_{2} \mathrm{Ti}$ and the hydride $\mathrm{U}_{2} \mathrm{TiH}_{3}$ have shown drastic differences between them related to:

1. The energy destabilizing effect of hydrogen on the intermetallic originating from a large anisotropic volume increase and subsequent Ti-U large separation and weakening of this bond.

2. The iono-covalent character brought by hydrogen through charge transfer $(\mathrm{Ti}, \mathrm{U}) \rightarrow \mathrm{H}$ and the bonding between them. 
3. U-U pairs arranging into 2D-like hexagons are at the origin of the largest cohesive energy of $\mathrm{U}_{2} \mathrm{Ti}$ versus $\mathrm{UTi}_{2}$ and $\mathrm{UT}$. They reinforced upon hydrogenation but a strong localization of out-of-plane $f$ orbitals create a magnetic instability in apparent violation of Hill criterion, leading to the development of a finite moments in a predicted ferromagnetic ground state. 
[1] A. Kianvash, I. R. Harris, J. Mater. Sci. 20 (1985) 682

[2] K. Asada, K. Ono, K. Yamaguchi, T. Yamamoto, A. Maekawa, S. Oe, M. Yamawaki, J. Alloys Compounds, 231 (1995) 780

[3] S.F. Matar Prog. Solid State Chem. 38 (2010) 1-37 (review).

[4] I. Halevy, S. Salhov, S. Zalkind, M. Brill, I. Yaar, J. Alloys and Compounds, 370 (2004) 59.

[5] S.F. Matar, A. Mavromaras, J. Solid State Chem., 149 (2000) 449.

[6] S.F. Matar, A.F. Al Alam, New Journal of Physics 10 (2008) 083013

[7] T. Yamamoto, M. Teshigawara, H. Kayano, N. Minakawa, S. Funahashi, J. Alloys and Compounds, 224 (1995) 36

[8] M.Z. Hasan, M.M. Hossain, M.S. Islam, F. Parvin, A.K.M.A. Islam, Comput. Mater. Sci., 63 (2012) 256

[9] P. Hohenberg, W. Kohn, Phys. Rev. B 136 (1964) 864.

W. Kohn, L.J. Sham, Phys. Rev. A 140 (1965) 1133.

[10] G. Kresse, J. Furthmüller, Phys. Rev. B 54 (1996) 11169.

[11] G. Kresse, J. Joubert, Phys. Rev. B 59 (1999) 1758.

[12] P. E. Blöchl, Phys. Rev. B 50 (1994) 17953.

[13] J. Perdew, K. Burke, M. Ernzerhof, Phys. Rev. Lett. 77 (1996) 3865

[14] D. M. Ceperley, B. J. Alder, Phys. Rev. Lett. 45 (1980) 566.

[15] W.H. Press, B.P. Flannery, S.A. Teukolsky, W.T. Vetterling, Numerical Recipes, Cambridge University Press, New York (1986).

[16] M. Methfessel, A. T. Paxton, Phys. Rev. B 40 (1989) 3616.

[17] R. Bader, Chem. Rev. 91 (1991) 893.

[18] A. R. Williams, J. Kübler, C. D. Gelatt, Phys. Rev. B 19 (1979) 6094.

[19] V. Eyert, The Augmented Spherical Wave Method - A Comprehensive Treatment, Lecture Notes in Physics, Springer, Heidelberg (2007).

[20] R. Hoffmann, Angew. Chem. Int. Ed. Engl. 26 (1987) 846.

[21] S.F. Matar, Computational Mater. Sci. 69, (2013) 424.

[22] F. Birch, Phys. Rev. 71 (1947) 809.

[23] P. Mohn, Magnetism in the solid state - An introduction, Springer Series, in: Solid-State Sciences. M. Cardona, P. Fulde, K. von Klitzing, R. Merlin, H.J. Queisser, H. Störmer (Eds.). Springer, Heidelberg (2003

[24] Hill 1970 Plutonium and Other Actinides ed. WN Miner (New York: Mat. Soc. Aime). 
Table 1. Calculated and (experimental) crystal structure data of $\mathrm{U}_{2} \mathrm{Ti}$ and its hydrogenated compounds studied in this work.

\begin{tabular}{|c|c|c|c|}
\hline$P 6 / m m m$ & $\mathrm{U}_{2} \mathrm{Ti}$ & $\begin{array}{l}\mathrm{U}_{2} \mathrm{TiH}_{3} \text { Hypo. } 1 \mathrm{H} \text { at } 3 f \\
(1 / 21 / 20 ; 1 / 200 ; 01 / 20)\end{array}$ & $\begin{array}{l}\mathrm{U}_{2} \mathrm{TiH}_{3} \mathrm{Hypo.} 2 \mathrm{H} \text { at } \\
3 g(1 / 201 / 2 ; 0 \quad 1 / 21 / 2 ; 1 / 21 / 21 / 2)\end{array}$ \\
\hline$a(\AA)$ & $4.77(4.828)$ & 4.653 & 5.967 \\
\hline$c / a$ & $0.59(0.59)$ & 0.79 & 0.46 \\
\hline$V\left(\AA^{3}\right)$ & $55.45(57.50)$ & 68.92 & 84.6 \\
\hline $\mathrm{d}_{\mathrm{Ti}-\mathrm{U}}$ & 3.09 & 3.25 & ---- \\
\hline$d_{U-U}$ & 2.75 & 2.70 & 3.44 \\
\hline$d_{\mathrm{Ti}-\mathrm{H}}$ & & ---- & ---- \\
\hline$d_{U-H}$ & & 2.27 & 1.72 \\
\hline $\begin{array}{l}\text { Total } \\
\text { energy } \\
\text { eV/FU }\end{array}$ & -30.59 & -40.12 & -33.82 \\
\hline $\begin{array}{l}\text { Cohesive } \\
\text { energy } \\
\text { eV/FU }\end{array}$ & -1.382 & -0.507 & +5.820 \\
\hline
\end{tabular}




\section{Figures captions}

Fig. 1: $\mathrm{U}_{2}$ Ti structure and its hydrogenated compound $\mathrm{U}_{2} \mathrm{TiH}_{3}$ with $\mathrm{H}$ at ( $3 f$ ) Wyckoff positions in $P 6 / \mathrm{mmm}$ space group. Projection onto $a, b$ plane highlights the U6 honeycomb -like arrangement of uranium at $z=1 / 2$.

Fig. 2: Energy volume curves of $\mathrm{U}_{2} \mathrm{Ti}$ (top) and $\mathrm{U}_{2} \mathrm{TiH}_{3}$ (bottom) and parameters of quadratic fit from the Birch EOS, given in the inserts.

Fig. 3: Non magnetic calculations. Site projected density of states $\mathrm{DOS} \mathrm{U}_{2} \mathrm{Ti}$ (top) and $\mathrm{U}_{2} \mathrm{TiH}_{3}$ (bottom).

Fig. 4: Magnetic calculations spin-only. Site and spin projected DOS in $\mathrm{U}_{2} \mathrm{Ti}$ (top) and $\mathrm{U}_{2} \mathrm{TiH}_{3}$ (bottom).

Fig. 5: Non magnetic calculations. Chemical bonding for metal-metal interactions in $\mathrm{U}_{2} \mathrm{Ti}$ (top) and $\mathrm{U}_{2} \mathrm{TiH}_{3}$ (bottom).

Fig. 6: Non magnetic calculations. Chemical bonding for metal-hydrogen interactions in $\mathrm{U}_{2} \mathrm{TiH}_{3}$. 

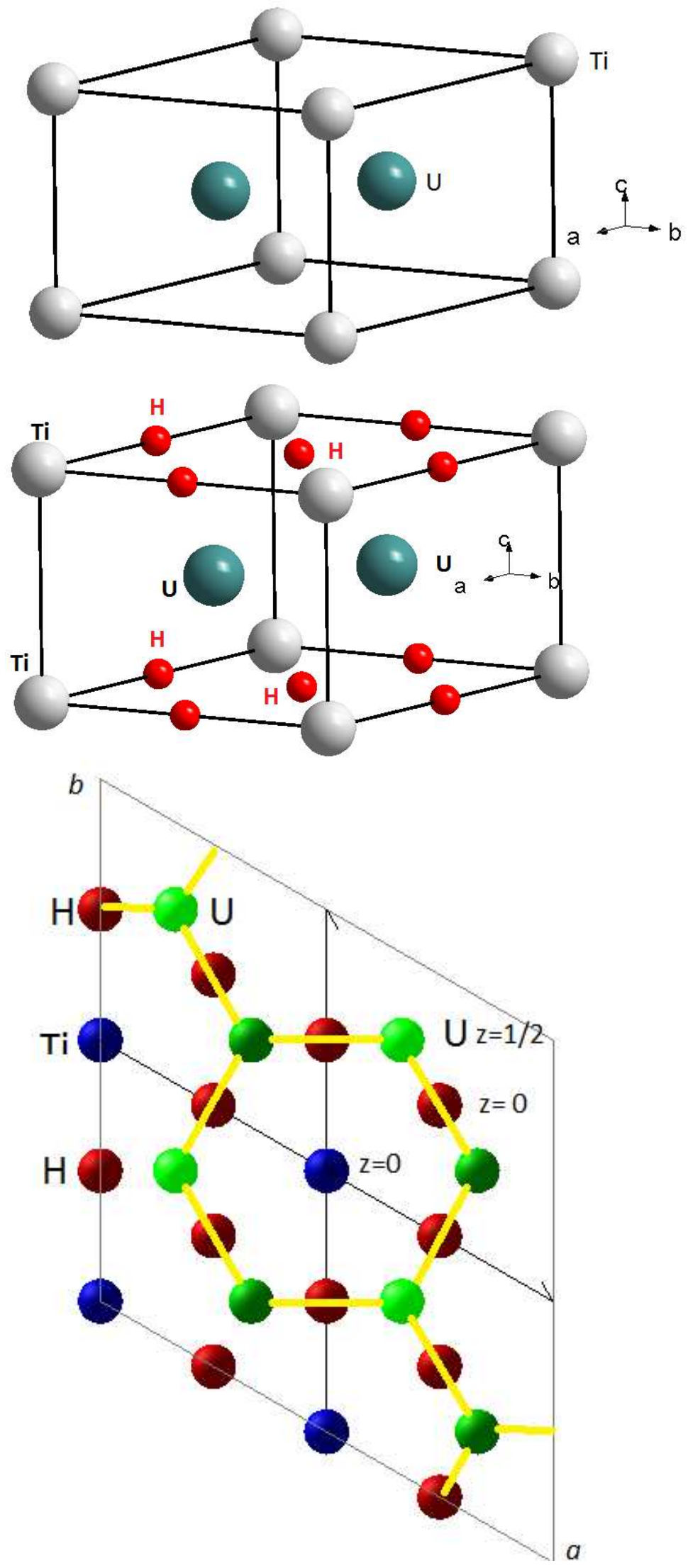

Fig.1 

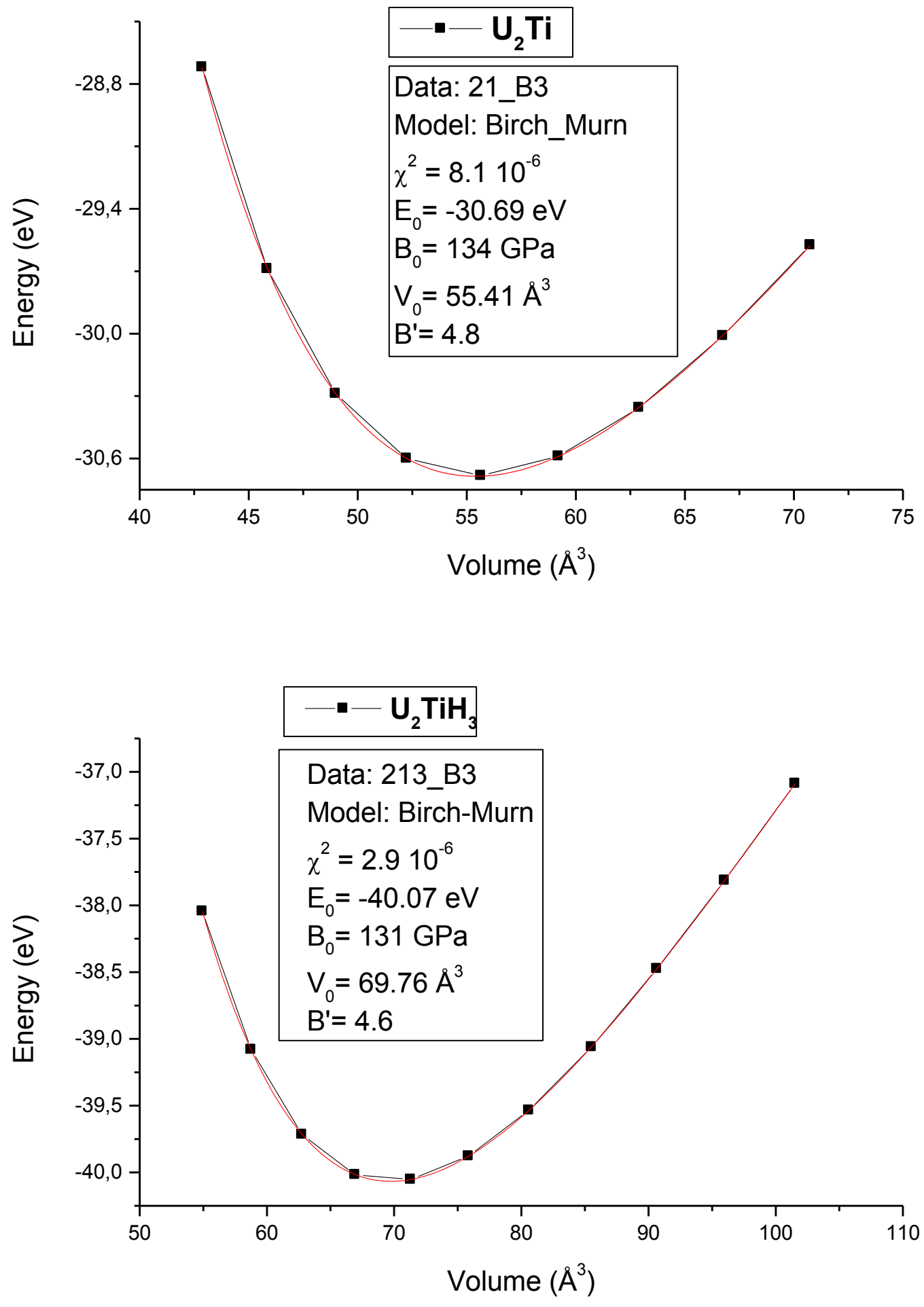

Fig. 2 

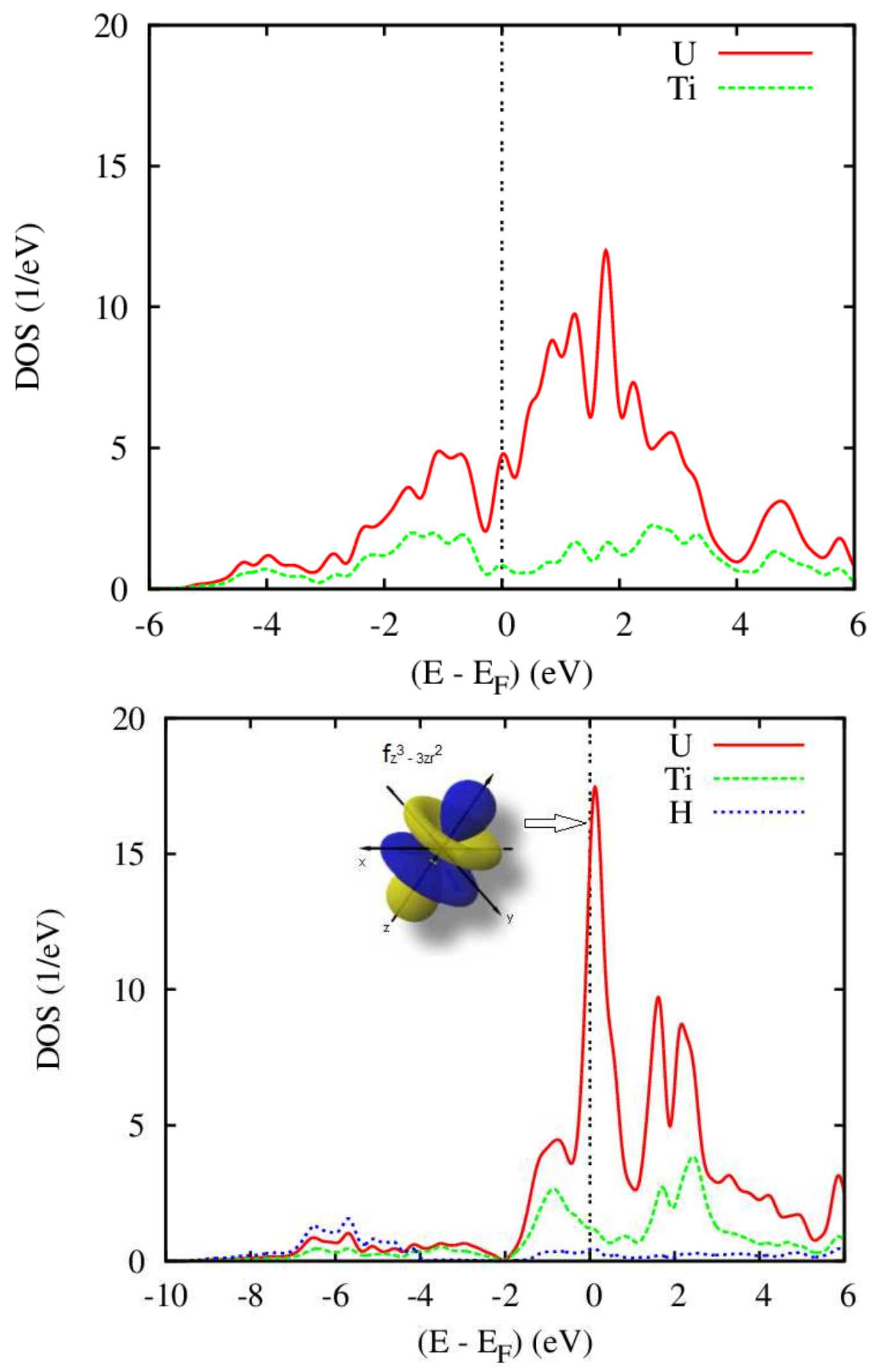

Fig. 3 

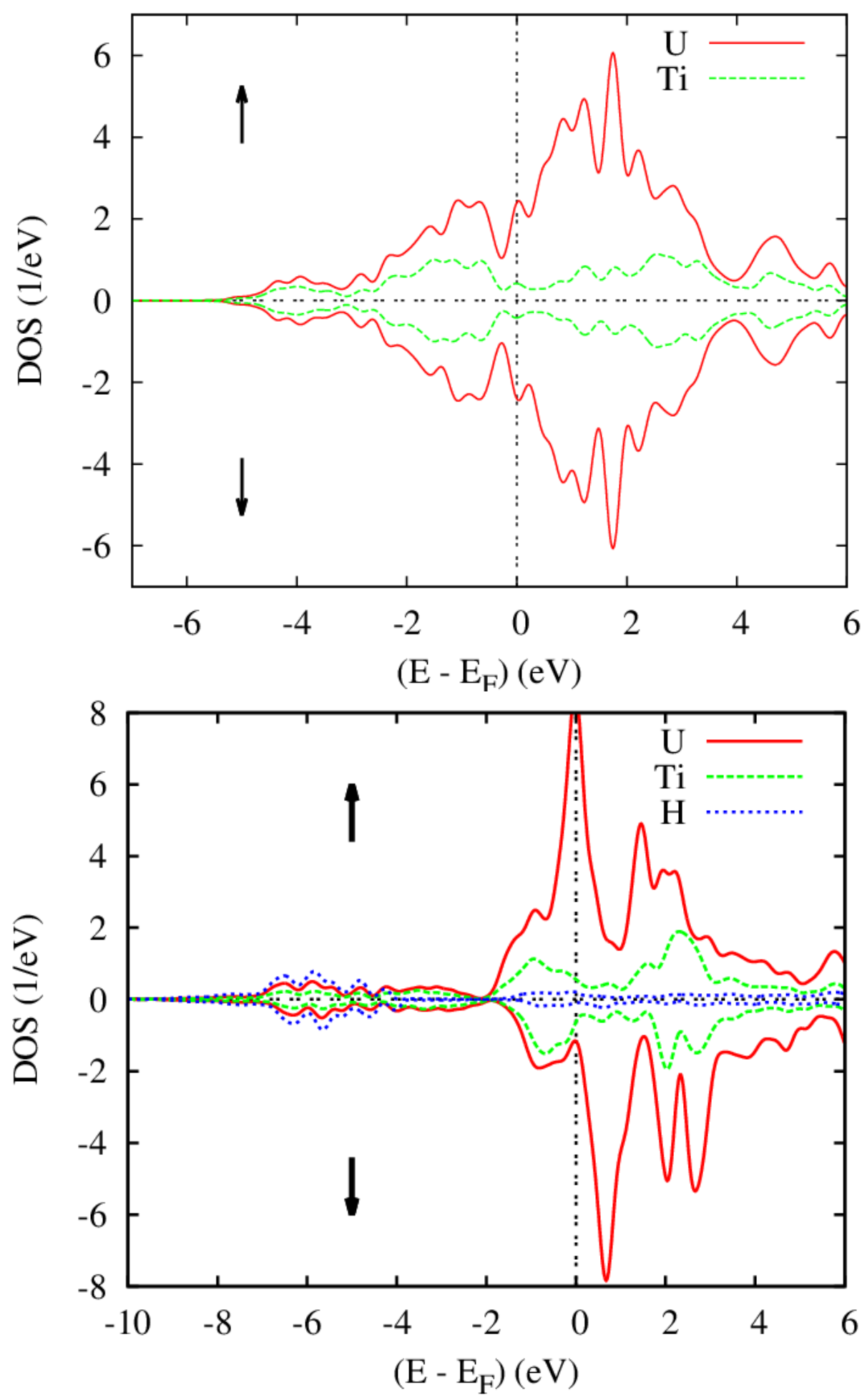

Fig.4 

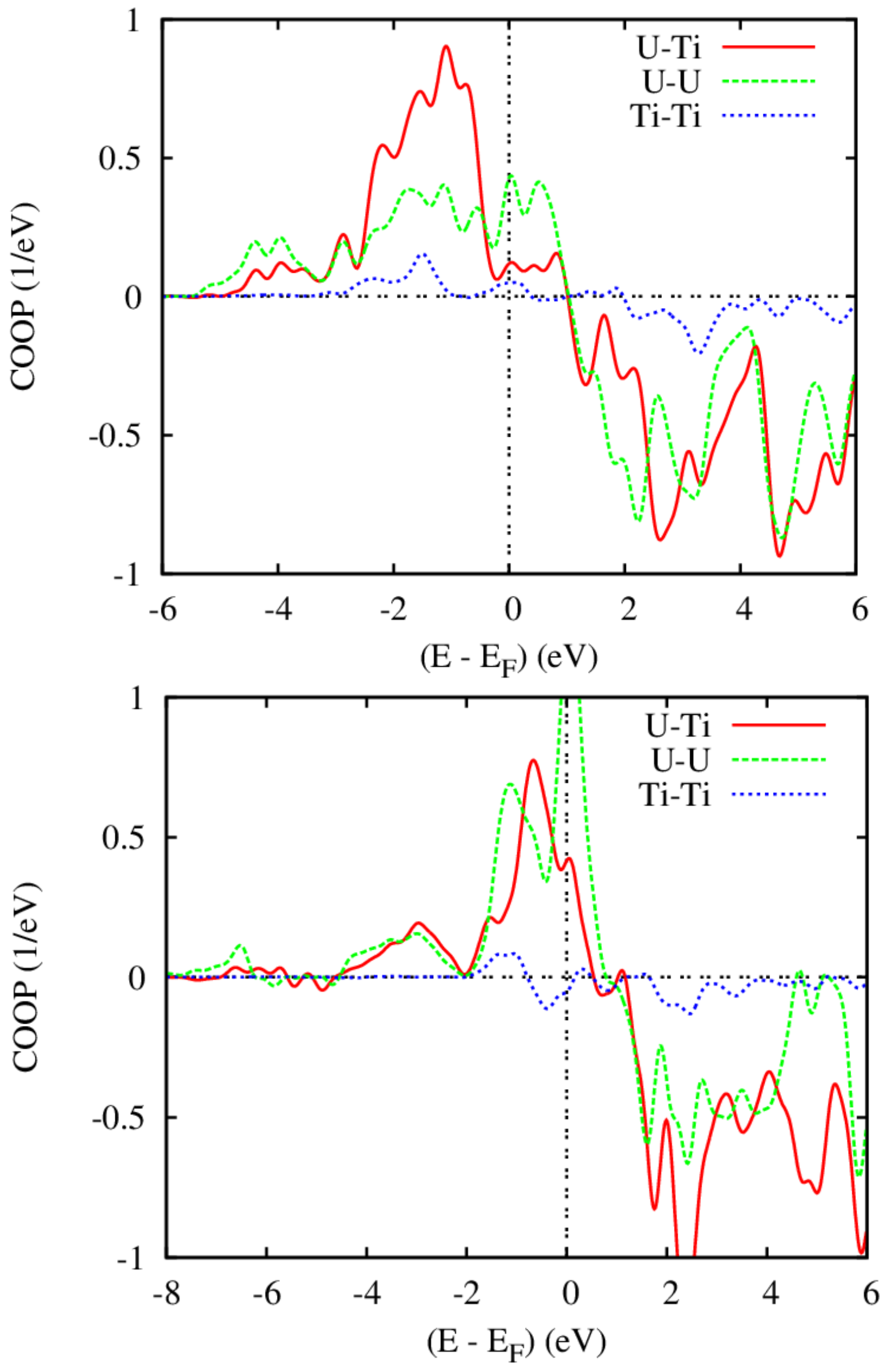

Fig. 5 


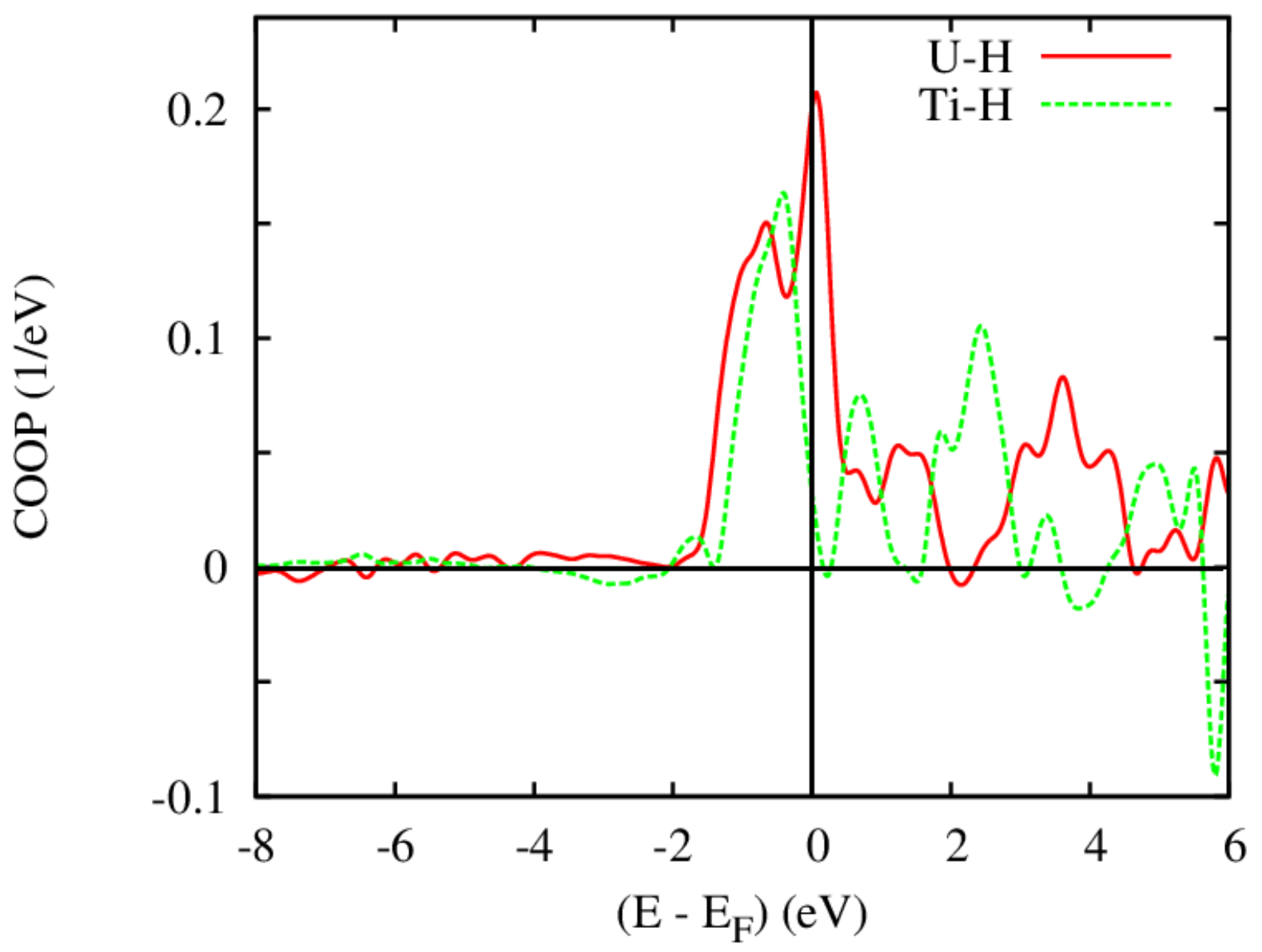

Fig. 6 